\title{
High performing SPS based on native NIR-emitting single colour centers in diamond
}

\author{
D. Gatto Monticone ${ }^{a, b, c}$, P. Traina ${ }^{d}$, E. Moreva ${ }^{d}$, J. Forneris ${ }^{a, b, c}$, M. Levi $^{a}$, G. Brida ${ }^{d}$, I. P.

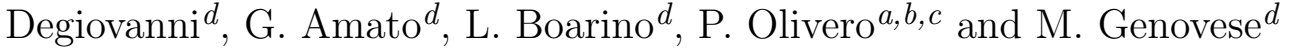 \\ ${ }^{a}$ Physics Department and NIS Centre of Excellence - University of Torino,Torino,Italy; \\ ${ }^{b}$ Istituto Nazionale di Fisica Nucleare (INFN) Sez. Torino,Torino,Italy; \\ ${ }^{c}$ Consorzio Nazionale Interuniversitario per le Scienze Fisiche della Materia (CNISM) - sez. \\ Torino, Italy; \\ ${ }^{d}$ Istituto Nazionale di Ricerca Metrologica (INRIM), Strada delle cacce 91, Torino, Italy.
}

\begin{abstract}
Single-photon sources (SPS) play a key-role in many applications, spanning from quantum metrology, to quantum information and to the foundations of quantum mechanics. Even if an ideal SPS (i. e. emitting indistinguishable, "on-demand" single photons, at an arbitrarily fast repetition rate) is far to be realized due to real-world deviations from the ideality, much effort is currently devoted to improving the performance of real sources. With regards to the emission probability, it appears natural to employ sources that are in principle deterministic in the singlephoton emission (single quantum emitters such as single atoms, ions, molecules, quantum dots, or color centers in diamond) as opposed to probabilistic ones (usually heralded SPS based on parametric down-conversion). We present an overview of our latest results concerning a work-in-progress NIR pulsed single photon source based on single quantum emitters (color centers in diamond) exploiting recently reported centers. They are particularly interesting because of the narrow emission line (tipically less than $5 \mathrm{~nm}$ ), the shorter excited state lifetime with respect to NV centres (1 - 2 ns compared to $12 \mathrm{~ns}$, allowing a ten-fold photon emission rate upon saturation) and the polarized emission.
\end{abstract}

Keywords: Single-photon source, confocal microscopy, diamond

\section{INTRODUCTION}

One of the open issues of the state of the art of quantum technology is the realisation of reliable, almost ideal Single-photon sources (SPS) and their optimization. Ideally a SPS should have three characteristics: it should be on demand (the emission time should be arbitrarily defined by the user, possibly with arbitrarily high rate), the probability of emitting zero or more than one photon in a proper time window should be zero, and subsequent photons should be indistinguishable. This goal is of course asymptotical and could not be likely met in the

\footnotetext{
Further author information: (Send correspondence to P. Traina)

P. Traina: E-mail: p.traina@inrim.it, Telephone: +39 (0)11 3919246

D. Gatto Monticone: monticon@to.infn.it

E. Moreva: e.moreva@inrim.it

J. Forneris: jacopo.forneris@unito.it

M. Levi: mattialevi@gmail.com

K. Katamadze: K.G.Katamadze@gmail.com

G. Brida: g.brida@inrim.it

I. P. Degiovanni: i.degiovanni@inrim.it

E. Enrico: e.enrico@inrim.it

G. Amato: g.amato@inrim.it

L. Boarino: 1.boarino@inrim.it

P. Olivero: paolo.olivero@unito.it

M. Genovese: m.genovese@inrim.it
} 
real world if not by approximation, but a great effort ${ }^{1,2}$ is spent in the quantum optics community in order to improve the current implementations, since the generation of a true single photon state is a fundamental tool in several applications spanning from quantum metrology, ${ }^{3-6}$ quantum information, ${ }^{7,8}$ and in the testing of the foundations of quantum mechanics. ${ }^{9-11}$

While, on the one hand, to achieve fully deterministic single-photon generation one could expect to be more convenient to employ sources based on intrinsically deterministic emitters (single atoms, ions, molecules, ${ }^{12}$ quantum dots, ${ }^{13}$ or color centers in diamond), behaving essentially as two level systems, on the other hand, due to the practical non-unit extraction efficiency (caused by internal reflection in the sample and coupling issues) generally affecting them, at the moment the probabilistic sources, most notably heralded sources based on parametric down-conversion, are the most suitable candidate for the best SPS approximation and, practically represent the state of the art. ${ }^{14}$ Nonetheless, realising a reliable SPS based on deterministic emitters would be of great interest in terms of cost, scalability and possible industrial application. Furthermore, probabilistic sources are affected by a non-zero multi-photon component that represent an effective drawback in many application in quantum information and quantum communication applications. For these reasons, it is justified the current interest in improving the performance (extraction efficiency, emission rate, good approximation of single photon state, wavelength and linewidth) of this kind of sources.

In this sense, in the last few years a fruitful line of research has been addressed to the realisation of sources based on colour centres in diamond, the latter being a broadly transparent crystal in which several luminescent centres can be easily found or implanted, and, in particular, studying single nitrogen-vacancy $\left(\mathrm{NV}^{-}\right) \mathrm{centres}^{15-17}$ has found wide application in quantum communication and quantum information ${ }^{18-21}$ since they are widely available, have good quantum efficiency and they have well known behavior in terms of electronic transitions. ${ }^{22}$

Unfortunately $\mathrm{NV}^{-}$centres have some strong limitations effecting their performance as useful single-photon sources, basically due to phonon coupling, long lifetime and charge-state blinking, so the research started to focus on other type of defects with characteristic more appealing in terms of integration with quantum optics and photonic technologies. Since the existence of efficient colour centres emitting in the telecom range is still debated [19], the search for interesting emitters is oriented in the near-infrared (NIR) range, where the wellestablished family Si-based photo-detectors are more efficient, and many types of luminescent centres (among which the ones related to Silicon, Nickel, Chromium and Xenon impurities) in that range have been studied both in single crystal and isolated nanocrystal diamond. ${ }^{23-28}$

Here we present an overview of our work-in-progress addressed to the realisation of a NIR pulsed singlephoton source exploiting color centers in diamond, which is based on a recently reported family of emitters of more appealing properties with respect to $\mathrm{NV}^{-} \mathrm{s}$ in terms of monochromaticity, excited state lifetime, saturation emission rate, stability and polarisation characteristics. In particular, such NIR centres, observed in several diamonds (nanocrystals, cvd type Ib and IIa diamond, natural samples) emit the whole PL light mainly into the zero-phonon-line, which can be as sharp as few nanometers at room-temperature and falls in a region ranging from 730 to $800 \mathrm{~nm}$. The structure and origin of this defects are still debated and a more detailed discussion on this matter can be found elsewere. ${ }^{29}$ Here we will limit ourselves to the characterization of this centers as single-photon emitters.

\section{EXPERIMENTAL SETUP}

In order to observe single colour centres in diamond, a single-photon sensitive custom-made confocal microscope (shown in Fig. 1) was realized at the laboratories of the "quantum optics group" of Istituto Nazionale di Ricerca Metrologica (INRIM). Such system has the advantage, with respect to commercial ones, to be more versatile since light emitted from colour centres can be directly coupled to other optical systems for quantum optics experiments once the most performing source has been selected. The excitation light at $690 \mathrm{~nm}$, originating from a Picoquant (Picosecond-Pulsed or CW) Laser diode, is infinity-focussed by a 4x objective. Eventual fluorescence originated inside the fibre is removed by a band-pass filter. Then, the beam is sent at the dichroic mirror (Long-pass $>700 \mathrm{~nm}$ ) which reflects the excitation light inside the objective (Zeiss Plan NeoFluar 100x NA = 0.9 air or alternatively OLYMPUS $100 \mathrm{x}, \mathrm{NA}=1.3$, oil) which focuses on the point of interest inside the sample. The sample is mounted on a closed-loop XYZ piezo-stage, controlled by a computer, allowing nanometric-resolution 


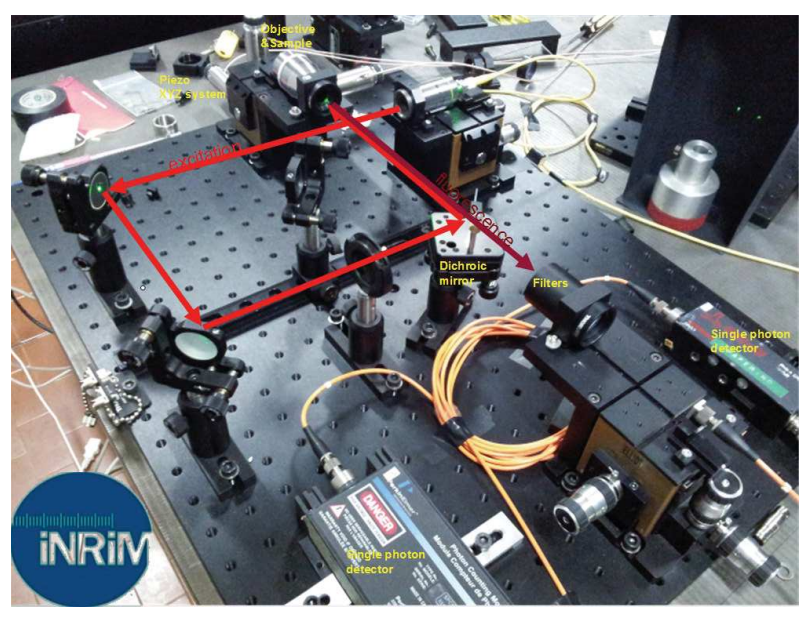

Figure 1. Experimental setup realising the single-photon-sensitive INRIM confocal microscope: the excitation light (pulsed or $\mathrm{cw}$ ) at $690 \mathrm{~nm}$ after being expanded by a $4 \mathrm{x}$ objective and filtered of eventual fluorescence generated inside the fiber, is addressed to a dichroic mirror (Long-pass $>700 \mathrm{~nm}$ ) which reflects it to the objective focusing on the sample. The fluorescence signal, after removing the residual pump component, is coupled to multi-mode fiber by an achromatic doublet ( $\mathrm{f}=100 \mathrm{~mm}$ ) and sent to HBT interferometer composed of a balanced fiber BS and two single-photon avalanche diodes (Excelitas SPCM-AQR) plus coincidence electronics (TAC and MCA). The samples, mounted on remotely controlled closed-loop XYZ piezo-stage allowing nanometric-resolution positioning and 100x100 $\mu \mathrm{m}$ scanning, are single-crystal and polycrystalline CVD diamonds produced by Element Six.

positioning and 100x100 $\mu \mathrm{m}$ scanning. The fluorescence light, collected by the same objective, is transmitted by the dichroic mirror and is long-pass filtered $(\lambda>730 \mathrm{~nm})$ in order to obtain a suitable attenuation $\left(>10^{12}\right)$ of the residual pump component. The signal is then focused by a $\mathrm{f}=100 \mathrm{~mm}$ achromatic doublet and coupled to a multi-mode optical fibre acting as the confocal pinhole. The fiber connects to a Hanbury-Brown and Twiss interferometer (HBT), consisting in an integrated balanced beam-splitter (not show in figure) plus two SinglePhoton Avalanche Diodes (SPAD) operating in Geiger mode. This configuration is widely used for second order autocorrelation function $\left(g^{(2)}(0)\right)$ measurement. Single detection events and coincidences are registered by the combined use of Time-to-Amplitude Converter (TAC) and Multi-Channel Analyser (MCA). The spectral analysis is performed by means of a single-grating monochromator (not shown in figure) with 1600 grooves $/ \mathrm{mm}$ blazed at $600 \mathrm{~nm}$ connected, when needed, to a SPAD.

\section{RESULTS AND DISCUSSION}

The setup was used to characterize several NIR centres from several single-crystal and polycrystalline CVD diamonds (type IIa) from Element Six, with nominal substitutional nitrogen concentrations being respectively less than $1 \mathrm{ppm}$ and $50 \mathrm{ppb}$. Annealing was performed in vacuum $\left(p<10^{-6} \mathrm{mbar}\right)$ at $1450^{\circ} \mathrm{C}$ for one hour. After annealing, the samples were treated ${ }^{29}$ to remove surface graphitization. Several maps of the samples were carried over by scanning their position via the XYZ piezo electric stage (remotely controlled by PC). Scanning resolution is typically $250 \mathrm{~nm} /$ pixel and the dwell time is $7 \mathrm{~ms}$ for a good signal/noise ratio. Fig. 2 (3) shows an example of imaging of one single-crystal (polycrystalline) sample. The picture shows a typical situation in which, in a region approximately 50x50 $\mu \mathrm{m}$, several centres can be observed. The distribution in depth seems to be constant, at least in the probing depth of our microscope that goes from the surface to about $20 \mu m$, at greater depths the spherical aberrations of the objective are too important to allow a detectable light collection from colour centres. There are many dots in the pictures that do not really correspond to single emitters: the first test that was used to check the nature of the suspected centers was the spectrum measurement via monochromator, to check that it was compatible with the known NIR centre emission. We found several different emission peaks, falling in a range between $730 \mathrm{~nm}$ to $800 \mathrm{~nm}$.

In particular, three families of emitters were observed, that can be classified by the emission wavelength: the 


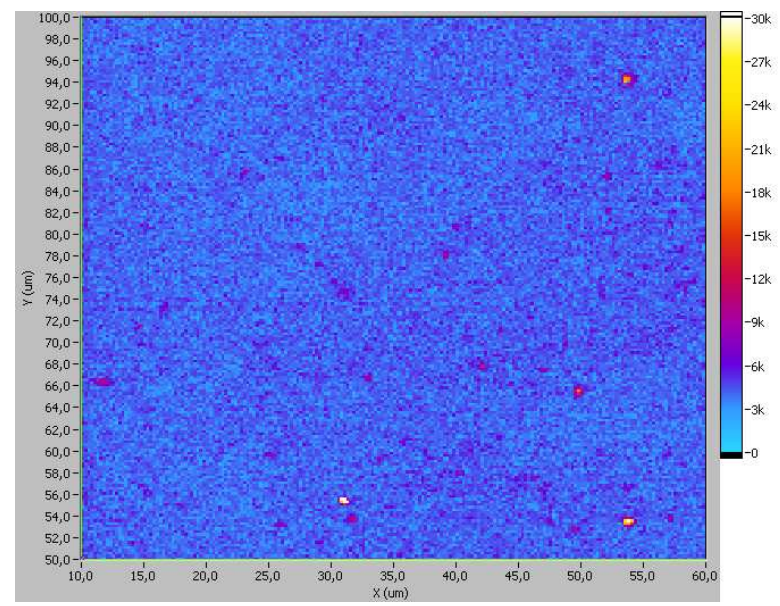

Figure 2. Example of a scan of a diamond sample (single crystal CVD by Element Six) by our confocal microscope. The intensity bar indicates emission rates.

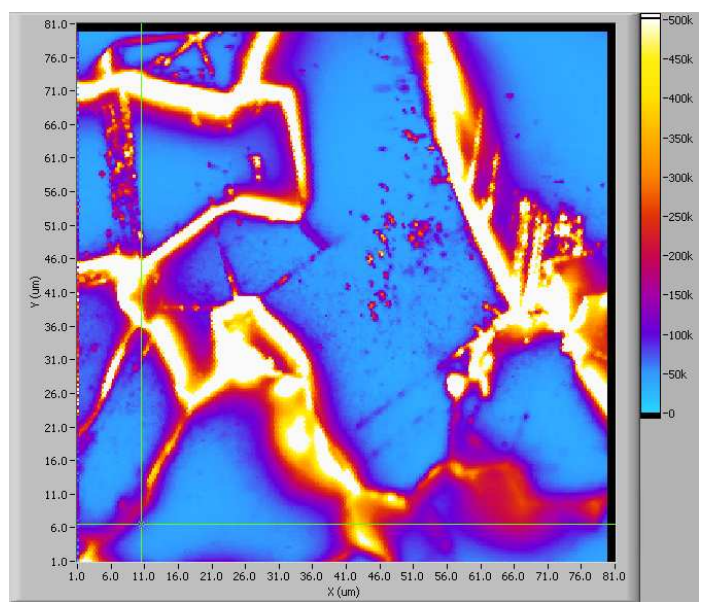

Figure 3. Example of a scan of a diamond sample (polycrystalline CVD by Element Six) by our confocal microscope. The intensity bar indicates emission rates. 


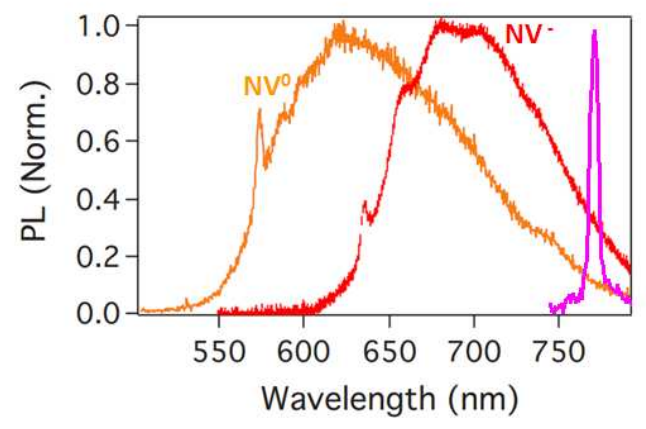

Figure 4. Normalised PL spectrum of the considered NIR centre compared to typical NV centres spectra. The ZPL line is at $772 \mathrm{~nm}$.

first family accounts emitters with ZPL around $755 \mathrm{~nm}$; a second family of centres with ZPL around $765 \mathrm{~nm}$; a third family of centres with ZPL around $775 \mathrm{~nm}$. Small shift of the ZPL may be attributed just by stress inside the crystal: birefringence that is commonly observed in such cvd samples supports this simple explanation.

It is also worth mentioning that most of the emitters suffer from photo-bleaching and/or photo blinking, other never bleaches but changes their count rate in time switching from two or more states characterized by a greater or lower emission rate. This phenomenon is a major drawback as for the possibility of realising a close to ideal SPS and it is observed in analogous experimental condition ${ }^{25}$ and it is more deeply treated elsewhere. ${ }^{29}$ A careful examination of the studied centres has been performed in order to select a reasonably stable one to be used as reliable single-photon source. An example of the emission spectrum of the selected NIR centre at $772 \mathrm{~nm}$ compared to typical spectra of NV centres is shown in Fig. 3, where it is evident the sharper linewidth, which is less than $5 \mathrm{~nm}$ (limited by the monochromator resolution) as opposed to $\approx 12 \mathrm{~nm}$, with respect to NV centres.

The centre under test has been tested in pulsed excitation (pulses of 68 ps with a repetition rate of $80 \mathrm{MHz}$ with an average power of $\approx 1 \mathrm{~mW}$ ), yielding the results shown in Fig. 4. Here one can observe the second order correlation function at the two detectors of the HBT in the form of an histogram of the arrival times of the photons on the STOP detector with respect to the times in which the START detector fired. The very low value of $g^{(2)}(0)$ reported $\left(g^{(2)}(0) \simeq 0.07\right.$, without blocking Raman line) shows the clear signature of the fact that the selected centre emits a good approximation a single-photon state.

Finally a measure of polarization on the emitter were carried over. In this case two measures were made, in the first the photon emission rate was measured with respect to the polarization angle of the pumping light, while in the second the polarization degree of the fluorescence light was measured. In both cases the dichroic mirror was replaced by a 50:50 beam splitter and its slightly polarization-dependent transmission and reflection changes were taken into account. The result is that the colour centres absorbs like an electric dipole polarized light along the $<110>$ direction (with respect to main crystallographic axis of diamond) and if light is orthogonallypolarized along this direction the fluorescence goes to zero, within the experimental uncertainty. In the same way, we measured dipole-like polarized fluorescence light, in direction orthogonal to the polarization axis for the absorption. The fact that the emission light is well polarized is important for a single photon source, because one can obtain photon of arbitrary polarization using waveplates with a good efficiency. For instance this is not the case of the NV centre, that emits light like two orthogonal dipoles and is then closer to a source of unpolarized light.

\section{CONCLUDING REMARKS}

We reported about the most recent results concerning our work-in-progress NIR pulsed single-photon source based on colour centres in diamond. The NIR-emitting single colour centres used (whose attribution is discussed 


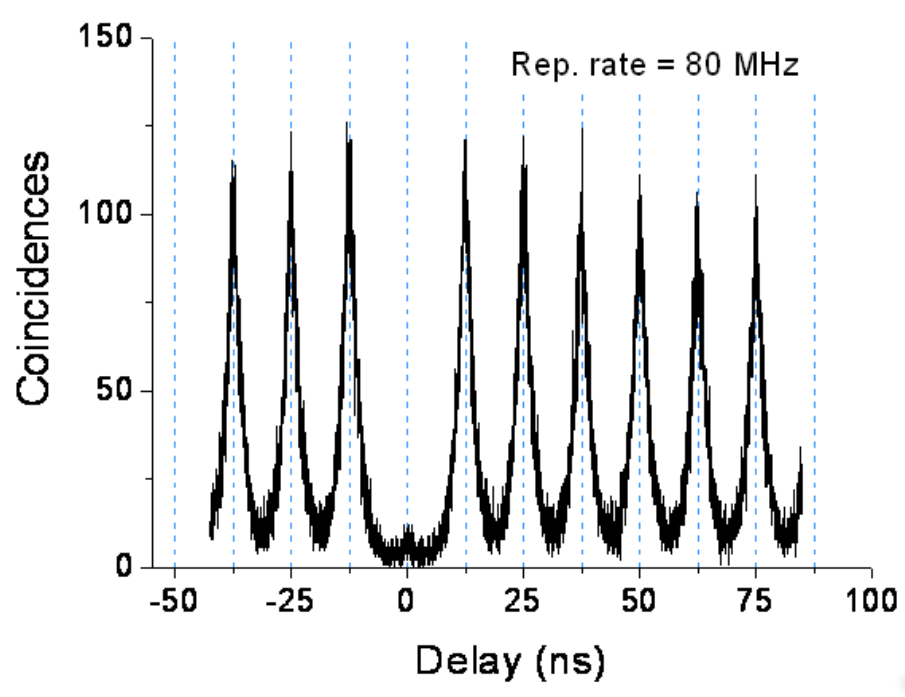

Figure 5. Preliminary measurement of second-order autocorrelation function $g^{(2)}(t)\left(g^{(2)}(0) \simeq 0.07\right)$ corresponding to the signal emitted by a NIR centre from single-crystal sample by Element Six in pulsed excitation (Raman line not blocked). For full information on the sample's specs and treatment, please see ref. ${ }^{29}$
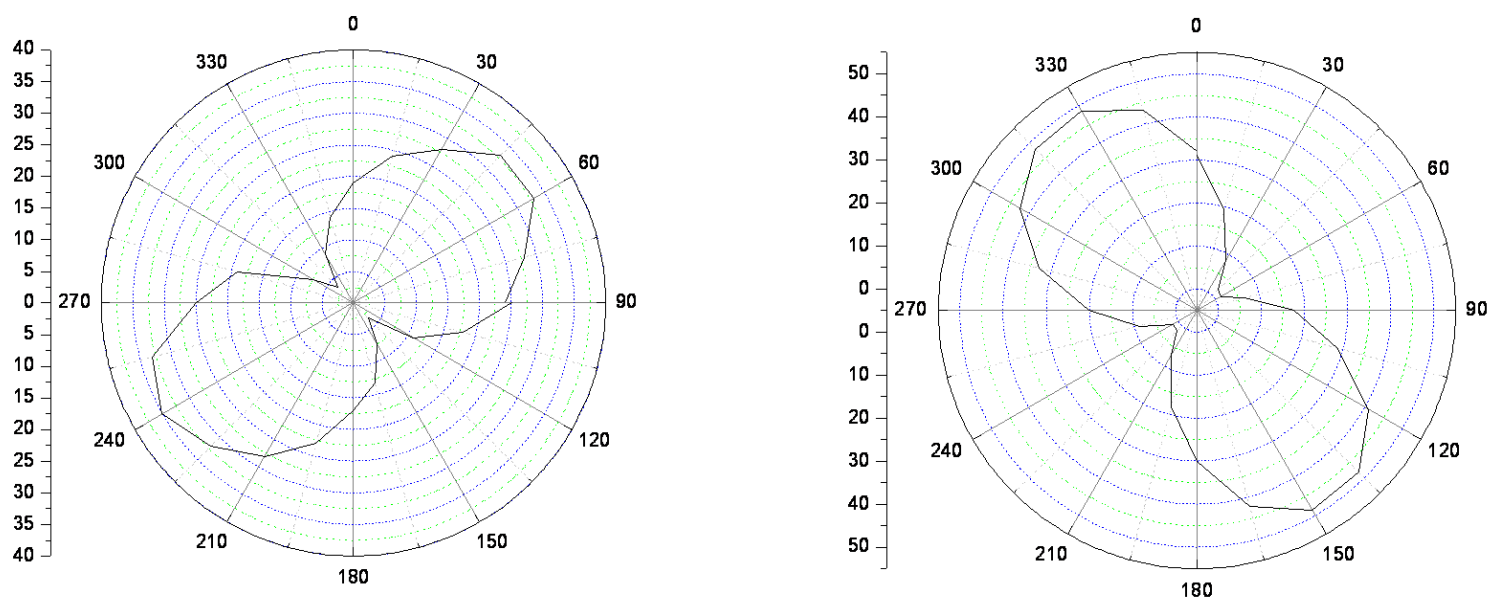

Figure 6. E

mission (right) and absorption (left) polarization measures for the centre at $772 \mathrm{~nm}$. $0^{\circ}$ is $<100>$ direction, the centre is seen by a [100] face on diamond, thus the dipole lies along $<110>$ directions. 
$\mathrm{in}^{29}$ ) were found on annealed optical-grade single-crystal and electronic-grade polycrystalline commercial CVD diamond samples and have been characterised by a custom made single-photon sensitive confocal microscope built at INRIM. This source exploits the properties highlighting the NIR centres as appealing single-photon emitters: narrow ZPL lines in an interesting spectral region, with low background and no measurable phonon sidebands, high emission rate with respect to NV centres, ${ }^{30,31}$ lifetime values comprised between $0.7 \mathrm{~ns}$ and $3 \mathrm{~ns}$, linearly polarized emission. Some encouraging preliminary results on the multi-photon component characterisation of the source obtained via HBT interferometer $\left(g^{(2)}(0) \simeq 0.07\right)$ have been shown, prompting to further investigation and application to quantum optics experiments.

\section{ACKNOWLEDGMENTS}

This work was funded by: FIRB "Future in Research 2010" project (CUP code: D11J11000450001) funded by the Italian Ministry for Teaching, University and Research (MIUR); EMRP projects "EXL02-SIQUTE" and "IND06-MIQC" (jointly funded by the EMRP participating countries within EURAMET and the European Union); "A.Di.NTech." project (CUP code: D15E13000130003) funded by University of Torino and Compagnia di San Paolo in the framework of the "Progetti di ricerca di Ateneo 2012" scheme; NATO SPS Project 984397; "Compagnia di San Paolo" project "Beyond classical limits in measurements by means of quantum correlations"; one-month DAAD 2011 type-1B grant "Ion implantation in diamond for applications in photonics" (application number: A/11/78148) funded by the German Academic Exchange Service.

\section{REFERENCES}

[1] S. Scheel, "Single-photon sources-an introduction," J. Mod. Opt. 56, pp. 141-160, 2009.

[2] M. D. Eisaman, J. Fan, A. Migdall, and S. V. Polyakov, "Single-photon sources and detectors," Rev. Sci. Instrum. 82, pp. 071101, 2011.

[3] G. Brida, M. Genovese, and M. Gramegna, "Twin-photon techniques for photo-detector calibration," Laser Phys. Lett. 3, pp. 115-123, 2006.

[4] S. V. Polyakov and A. L. Migdall, "Quantum radiometry," J. Mod. Opt. 56(9), pp. 1045-1052, 2009.

[5] J. C. Zwinkels, E. Ikonen, N. P. Fox, G. Ulm, and M. L. Rastello, "Photometry, radiometry and 'the candela': evolution in the classical and quantum world," Metrologia 47, R15, 2010.

[6] W. Schmunk, M. Rodenberger, S. Peters, H. Hofer, S. Kueck, "Radiometric calibration of single photon detectors by a single photon source based on NV-centers in diamond," J. Mod. Opt. 58 (14), pp. 1252-1259, 2011.

[7] R. Thew and N. Gisin, "Quantum communication," Nat. Photonics 1, pp. 165-171, 2007.

[8] J. L. O'Brien, A. Furusawa, and J. Vickovic, "Photonic quantum technologies," Nat. Photonics 3, pp. 687$695,2009$.

[9] M. Genovese, "Research on hidden variable theories: A review of recent progresses," Phys. Rep. 413, pp. 319396, 2005.

[10] G. Brida, I. P. Degiovanni, M. Genovese, V. Schettini, S. Polyakov, and A. Migdall, "Experimental test of nonclassicality for a single particle," Opt. Express 16, pp. 11750-11758, 2008.

[11] G. Brida, I. P. Degiovanni, M. Genovese, F. Piacentini, V. Schettini, N. Gisin, S. V. Polyakov, and A. Migdall, "Improved implementation of the AlickiVan Ryn nonclassicality test for a single particle using Si detectors," Phys. Rev. A 79, pp. 044102, 2009.

[12] B. Lounis and W. E. Moerner, "Single photons on demand from a single molecule at room temperature," Nature 407, pp. 491-493, 2000.

[13] Z. L. Yuan, B. E. Kardynal, R. M. Stevenson, A. J. Shields, C. J. Lobo, K. Cooper, N. S. Beattie, D. A. Ritchie, M. Pepper, "Electrically Driven Single-Photon Source," Science 295, pp. 102-105, 2002.

[14] G. Brida, I. P. Degiovanni, M. Genovese, F. Piacentini, P. Traina, A. Della Frera, A. Tosi, A. Bahgat Shehata, C. Scarcella, A. Gulinatti, M. Ghioni, S. V. Polyakov, A. Migdall and A. Giudice, "An extremely low-noise heralded single-photon source: A breakthrough for quantum technologies," Appl. Phys. Lett. 101 (22), pp. 221112-221112-4, 2012. 
[15] C. Kurtsiefer, S. Mayer, P. Zarda, and H. Weinfurter, "Stable Solid-State Source of Single Photons," Phys. Rev. Lett. 85 (2), pp. 290-293, 2000.

[16] A. Beveratos, R. Brouri, T. Gacoin, J. -P. Poizat, and P. Grangier, "Nonclassical radiation from diamond nanocrystals," Phys. Rev. A $\mathbf{6 4}$ pp. 061802, 2001.

[17] A. Beveratos, S. Khn,R. Brouri, T. Gacoin, J. -P. Poizat, and P. Grangier "Room temperature stable single-photon source," Eur. Phys. J. D 18 pp. 191-196, 2002.

[18] A. Beveratos, R. Brouri, T. Gacoin, A. Villing, J. -P. Poizat, and P. Grangier, "Single Photon Quantum Cryptography," Phys. Rev. Lett. 89 (18), pp. 187901, 2002.

[19] R. Allaume, F. Treussart, G. Messin, Y. Dumeige, J. -F. Roch, A. Beveratos, R. Brouri-Tualle, J. -P. Poizat, and P. Grangier, "Experimental open-air quantum key distribution with a single-photon source," New J. Phys. 6, pp. 92, 2004.

[20] L. Childress, J. M. Taylor, A. S. Sørensen, and M. D. Lukin, "Fault-Tolerant Quantum Communication Based on Solid-State Photon Emitters ," Phys. Rev. Lett. 96, pp. 070504, 2006.

[21] W. Schmunk, M. Gramegna, G. Brida, I. P. Degiovanni, M. Genovese, H. Hofer, S. Kück, L. Lolli, M. G. A. Paris, S. Peters, M. Rajteri, A. M. Racu, A. Ruschhaupt, E. Taralli, and P. Traina, "Photon number statistics of NV centre emission ," Metrologia 49, pp. S156-S160, 2012.

[22] V. Acosta and P. Hemmer, "Nitrogen-vacancy centers: Physics and applications," MRS Bull. 38, pp. 127130, 2013.

[23] C. Wang, C. Kurtsiefer, H. Weinfurter, and B. Burchard, "Single photon emission from SiV centres in diamond produced by ion implantation," J. Phys. B At. Mol. Opt. Phys. 39, pp. 37-41, 2006.

[24] E. Neu, C. Hepp, M. Hauschild, S. Gsell, M. Fischer, H. Sternschulte, D. Steinmller-Nethl, M. Schreck, and C. Becher, "Low-temperature investigations of single silicon vacancy colour centres in diamond," New J. Phys. 15, pp. 043005, 2013.

[25] D. Steinmetz, E. Neu, J. Meijer, W. Bolse, and C. Becher, "Single photon emitters based on Ni/Si related defects in single crystalline diamond," Appl. Phys. B 102, pp. 451-458, 2011.

[26] K. -M. C. Fu, C. Santori, P. E. Barclay, I. Aharonovich, S. Prawer, N. Meyer, A. M. Holm, and R. G. Beausoleil, "Coupling of nitrogen-vacancy centers in diamond to a GaP waveguide," Appl. Phys. Lett. 93, pp. 243112, 2008.

[27] I. Aharonovich, S. Castelletto, B. C. Johnson, J. C. McCallum, D. A. Simpson, A. D. Greentree, and S. Prawer "Chromium single-photon emitters in diamond fabricated by ion implantation," Phys. Rev. B 81, pp. 121201, 2010.

[28] Y. Deshko and A. A. Gorokhovsky, "Spectroscopy and micro-luminescence mapping of Xe-implanted defects in diamond," J. Low Temp. Phys. 36 (5), pp. 465-471, 2010.

[29] D. Gatto Monticone, P. Traina, E. Moreva, J. Forneris, P. Olivero, I. P. Degiovanni, F. Taccetti, L. Giuntini, G. Brida, G. Amato and M. Genovese, "Native NIR-emitting single colour centres in CVD diamond," arXiv:1403.4394

[30] R. Brouri, A. Beveratos, J. -P. Poizat, and P. Grangier, "Photon antibunching in the fluorescence of individual color centers in diamond," Opt. Lett. 25 (17), pp. 1294-1296, 2000.

[31] T. M. Babinec, B. J. M. Hausmann, M. Khan, Y. Zhang, J. R. Maze, P. R. Hemmer and M. Lonĉar, "A diamond nanowire single-photon source," Nat. Nanotechnol. 5, pp. 195-199, 2010. 